\title{
Thyroid Hormone Profile and Its Prognostic Impact on the Coronavirus Disease 2019 in Korean Patients
}

\author{
Jiyeon Ahn, Min Kyung Lee, Jae Hyuk Lee, Seo Young Sohn
}

Division of Endocrinology and Metabolism, Department of Internal Medicine, Myongji Hospital, Hanyang University College of Medicine, Goyang, Korea

Background: Data on the association between coronavirus disease 2019 (COVID-19) and thyroid have been reported, including overt thyrotoxicosis and suppression of thyroid function. We aimed to evaluate the thyroid hormone profile and its association with the prognosis of COVID-19 in Korean patients.

Methods: The clinical data of 119 patients with COVID-19, admitted in the Myongi Hospital, Goyang, South Korea, were retrospectively evaluated. The thyroid hormone profiles were analyzed and compared based on disease severity (non-severe disease vs. severe to critical disease). Clinical outcomes were analyzed according to the tertiles of thyroid hormones.

Results: Of the 119 patients, 76 (63.9\%) were euthyroid, and none presented with overt thyroid dysfunction. Non-thyroidal illness syndrome was the most common manifestation (18.5\%), followed by subclinical thyrotoxicosis (14.3\%) among patients with thyroid dysfunction. Thyroid stimulating hormone (TSH) and triiodothyronine (T3) levels were significantly lower in patients with severe to critical disease than in those with non-severe disease $(P<0.05)$. Patients in the lowest T3 tertile $(<0.77 \mathrm{ng} / \mathrm{mL})$ had higher rates of mechanical ventilation, intensive care unit admission, and death than those in the middle and highest $(>1.00 \mathrm{ng} / \mathrm{mL}) \mathrm{T} 3$ tertiles $(P<0.05)$. COVID-19 patients in the lowest T3 tertile were independently associated with mortality (hazard ratio, 5.27 ; $95 \%$ confidence interval, 1.09 to $25.32 ; P=0.038$ ) compared with those in the highest T3 tertile.

Conclusion: Thyroid dysfunction is common in COVID-19 patients. Changes in serum TSH and T3 levels may be important markers of disease severity in COVID-19. Decreased T3 levels may have a prognostic significance in COVID-19 related outcome.

Keywords: COVID-19; Thyroid hormones; Triiodothyronine; Euthyroid sick syndromes; Mortality

\section{INTRODUCTION}

Since the outbreak of the coronavirus disease 2019 (COVID-19) in December 2019, COVID-19 has spread rapidly worldwide and has been declared as a pandemic by the World Health Organization in March 2020 [1]. The causative agent of COVID-19 is severe acute respiratory syndrome coronavirus 2 (SARSCoV-2) [2], which can invade human cells through the angioten-

Received: 13 May 2021, Revised: 1 July 2021, Accepted: 16 July 2021

Corresponding author: Seo Young Sohn

Division of Endocrinology and Metabolism, Department of Internal Medicine, Myongji Hospital, Hanyang University College of Medicine, 55 Hwasu-ro 14beon-gil, Deokyang-gu, Goyang 10475, Korea Tel: +82-31-810-5409, Fax: +82-31-969-0500, E-mail: drsohnsy@gmail.com sin-converting enzyme 2 (ACE2) receptor. The thyroid gland shows high ACE2 expression levels, making the thyroid gland a possible target of SARS-CoV-2 [3]. Several studies have reported the development of subacute thyroiditis after COVID-19 [48]. In contrast, other investigators showed that the acute effect of COVD-19 on thyroid function was more consistent with a nonthyroidal illness syndrome (NTIS) pattern, characterized by mild reduction in triiodothyronine (T3) and/or thyroxine (T4) levels

Copyright $(\odot) 2021$ Korean Endocrine Society

This is an Open Access article distributed under the terms of the Creative Commons Attribution Non-Commercial License (https://creativecommons.org/ licenses/by-nc/4.0/) which permits unrestricted non-commercial use, distribution, and reproduction in any medium, provided the original work is properly cited. 
without increasing the thyroid stimulating hormone (TSH) levels [9-14]. NTIS is considered as an adaptive response to acute illness, occurring during the fasting state in healthy individuals, as well as to other systemic diseases, including cardiovascular and infectious diseases [15-17]. Previous studies have shown that low T3 concentrations are usually associated with an adverse prognosis in patients with critical illness $[18,19]$. However, other studies have shown that decreased TSH concentrations were independently associated with mortality in patients with liver failure [20] or older adults in the community [21].

In this retrospective cohort study, we aimed to analyze the characteristics of thyroid hormone levels and evaluate their association with prognosis of COVID-19.

\section{METHODS}

\section{Study design and population}

This retrospective cohort study included COVID-19 patients who were admitted in Myongji Hospital in Goyang, Republic of Korea, from January 2020 to March 2021. The hospital in this study operates nationally designated negative-pressure isolation beds and treats COVID-19 patients separately from general patients [22]. In addition, five intensive care units (ICUs) and one special ward were designated for COVID-19 patients. The diagnosis of COVID-19 was confirmed when the results of reverse transcription polymerase chain reaction assay of upper respiratory specimens (nasopharyngeal and oropharyngeal swabs), with or without sputum, were positive for SARS-CoV-2. After exclusion of patients with a previous history of thyroid dysfunction, thyroid surgery, use of exogenous steroids, pregnancy, and absence of thyroid function tests, 119 patients were included in the current study.

The study protocol was approved by the Institutional Review Board (IRB) of Myongji Hospital, and the requirement for informed consent was waived by the ethics board (IRB file no. 2021-05-010) due to the retrospective and observational nature of this study.

\section{Data collection}

Demographic information, laboratory data, and clinical data were retrospectively collected from the electronic medical records. Blood samples were obtained from the patients on the first day of admission and used for complete blood count, biochemical tests, thyroid hormone test, and high-sensitivity C-reactive protein (hs-CRP) test, regardless of the fasting state.

\section{Thyroid function test}

Thyroid hormone profiles, including TSH, T3, and free T4 (FT4) levels, were measured by quantitative chemiluminescent immunoassays using the Atellica IM analyzer (Siemens Healthineers, Erlangen, Germany). The assay showed TSH, T3, and FT4 levels of $0.008-150 \mathrm{mIU} / \mathrm{L}, 0.10-8.00 \mathrm{ng} / \mathrm{mL}$, and $0.1-$ $12.0 \mathrm{ng} / \mathrm{dL}$, respectively. The TSH assay was designed to have within-laboratory precision of $\leq 0.0032 \mathrm{mIU} / \mathrm{L}$ standard deviation (SD) for samples $<0.020 \mathrm{mIU} / \mathrm{L}, \leq 16 \%$ coefficient of variation $(\mathrm{CV})$ for samples ranging from 0.020 to $0.299 \mathrm{mIU} / \mathrm{L}$, $\leq 8 \% \mathrm{CV}$ for samples ranging from 0.300 to $90.00 \mathrm{mIU} / \mathrm{L}$, and $\leq 10 \% \mathrm{CV}$ for samples $>90.00 \mathrm{mIU} / \mathrm{L}$. The T3 assay was designed to have within-laboratory precision of $\leq 0.08 \mathrm{ng} / \mathrm{mL} \mathrm{SD}$ for samples $<0.60 \mathrm{ng} / \mathrm{mL}, \leq 13 \% \mathrm{CV}$ for samples ranging from 0.60 to $0.80 \mathrm{ng} / \mathrm{mL}, \geq 11 \% \mathrm{CV}$ for samples ranging from 0.80 to $1.80 \mathrm{ng} / \mathrm{mL}$, and $\leq 10 \% \mathrm{CV}$ for samples $>1.80 \mathrm{ng} / \mathrm{mL}$. The FT4 assay was designed to have within laboratory precision of $\leq 0.03 \mathrm{SD}$ for samples $<0.5 \mathrm{ng} / \mathrm{dL}, \leq 8.0 \% \mathrm{CV}$ for samples ranging from 0.5 to $1.0 \mathrm{ng} / \mathrm{dL}$, and $<6.0 \% \mathrm{CV}$ for samples $>1.0$ $\mathrm{ng} / \mathrm{dL}$. The reference ranges of TSH, T3, and FT4 were 0.55 $4.78 \mathrm{mIU} / \mathrm{L}, 0.6-1.8 \mathrm{ng} / \mathrm{mL}$, and $0.89-1.76 \mathrm{ng} / \mathrm{dL}$, respectively, as recommended by the kit provider.

\section{Definition of condition}

The diagnostic categories for thyroid function were as follows: (1) euthyroid (normal TSH with normal T3 and FT4 levels); (2) overt thyrotoxicosis (decreased TSH values and increased FT4 levels); (3) overt hypothyroidism (increased TSH values and decreased serum FT4); (4) subclinical hypothyroidism (increased TSH values with normal FT4 levels); (5) subclinical thyrotoxicosis (decreased TSH values but normal FT4 levels); and (6) NTIS (decreased T3 level only, or decreased T3, TSH, and/or FT4 levels).

The severity of COVID-19 was divided into non-severe and severe to critical [23]. Severe illness was defined as COVID-19 with respiratory rate of $\geq 30$ per minute, oxygen saturation of $\leq$ $93 \%$, or $\mathrm{PaO}_{2} / \mathrm{FiO}_{2}$ of $\leq 300 \mathrm{~mm} \mathrm{Hg}$ on admission. Patients who developed respiratory failure requiring mechanical ventilation, septic shock, and/or organ failure requiring monitoring and treatment in the ICU were classified as having critical illness. Patients without the abovementioned conditions were classified as having non-severe illnesses.

\section{Statistical analysis}

Statistical analyses were performed using SPSS version 22 (IBM Corp., Armonk, NY, USA). Continuous variables with normal 
distribution were presented as mean $\pm \mathrm{SD}$ and were compared using $t$ tests. Variables with skewed distribution were presented as medians with interquartile ranges and were compared using the Mann-Whitney $U$ tests. Categorical data were expressed as numbers with percentages and were compared using chi-square tests. The correlations between thyroid hormone and hs-CRP or white blood cell (WBC) counts were determined by calculating the Pearson correlation coefficient. The Cochran-Armitage test for trend was used to analyze the association between thyroid hormone tertiles (lowest, middle, and highest tertile of TSH, T3, and FT4 levels) and clinical outcomes of patients. The KaplanMeier curves were used to describe the cumulative survival of patients according to tertiles of T3 levels. Follow-up was started on the admission date and was censored on the date of death or the last day of follow-up (April 15, 2021). The Cox proportional hazard model was used, and hazard ratios (HRs) with the corresponding 95\% confidence intervals (CIs) were computed to examine the independent association between T3 tertiles and risk of mortality.

\section{RESULTS}

\section{Baseline characteristics}

The baseline characteristics of all COVID-19 patients are shown in Table 1. These characteristics were compared between survivors and non-survivors. The patients' mean age was $64.3 \pm$ 16.8 years, and $52.1 \%$ of patients were men. Hypertension was the most prevalent comorbidity $(52.1 \%)$, followed by diabetes

Table 1. Clinical Characteristics of 119 Patients with COVID-19

\begin{tabular}{|c|c|c|c|c|}
\hline Characteristic & Total $(n=119)$ & Survivors $(n=85)$ & Non-survivors $(n=34)$ & $P$ value \\
\hline Age, yr & $64.3 \pm 16.8$ & $59.7 \pm 17.0$ & $75.7 \pm 9.6$ & $<0.001$ \\
\hline Sex, male & $62(52.1)$ & $43(50.6)$ & $19(55.9)$ & 0.601 \\
\hline \multicolumn{5}{|l|}{ Comorbidities } \\
\hline Diabetes & $36(30.3)$ & $21(24.7)$ & $15(44.1)$ & 0.037 \\
\hline HTN & $62(52.1)$ & $37(43.5)$ & $25(73.5)$ & 0.003 \\
\hline Ischemic heart disease & $11(9.2)$ & $7(8.2)$ & $4(11.8)$ & 0.548 \\
\hline COPD/asthma & $10(8.4)$ & $6(7.1)$ & $4(11.8)$ & 0.403 \\
\hline Malignancy & $23(19.3)$ & $18(21.2)$ & $5(14.7)$ & 0.419 \\
\hline Stoke/cerebral hemorrhage & $11(9.2)$ & $2(5.9)$ & $9(10.6)$ & 0.423 \\
\hline \multicolumn{5}{|l|}{ Symptoms } \\
\hline Symptomatic & $107(89.9)$ & $75(88.2)$ & $32(94.1)$ & 0.336 \\
\hline Fever & $65(54.6)$ & $48(56.5)$ & $17(50)$ & 0.522 \\
\hline Cough/sputum & $61(51.3)$ & $46(54.1)$ & $15(44.1)$ & 0.324 \\
\hline Rhinorrhea & $4(3.4)$ & $3(3.5)$ & $1(2.9)$ & 0.999 \\
\hline Shortness of breath & $48(40.3)$ & $22(25.9)$ & $26(76.5)$ & $<0.001$ \\
\hline Neck pain & $9(7.6)$ & $8(9.4)$ & $1(2.9)$ & 0.443 \\
\hline Headache & $10(8.4)$ & $8(9.4)$ & $2(5.9)$ & 0.722 \\
\hline Nausea/vomiting & $10(8.4)$ & $7(8.2)$ & $3(8.8)$ & 0.999 \\
\hline Diarrhea & $16(13.4)$ & $13(15.3)$ & $3(8.8)$ & 0.553 \\
\hline Anosmia/ageusia & $5(4.2)$ & $5(5.9)$ & 0 & 0.320 \\
\hline \multicolumn{5}{|l|}{ Initial laboratory finding } \\
\hline WBC count, $\times 10^{9} / \mathrm{L}$ & $7.73 \pm 5.96$ & $6.80 \pm 3.50$ & $10.1 \pm 9.36$ & 0.055 \\
\hline C-reactive protein, mg/dL & $7.3 \pm 7.2$ & $5.8 \pm 5.9$ & $11.1 \pm 8.6$ & 0.002 \\
\hline Mean GFR, $\mathrm{mL} / \mathrm{min} / 1.73 \mathrm{~m}^{2}$ & $90.0 \pm 25.2$ & $95.2 \pm 25.0$ & $76.7 \pm 20.1$ & $<0.001$ \\
\hline GFR $<60 \mathrm{~mL} / \mathrm{min} / 1.73 \mathrm{~m}^{2}$ & $14(11.8)$ & $8(9.4)$ & $6(17.6)$ & $<0.001$ \\
\hline
\end{tabular}

Values are expressed as mean \pm standard deviation or number $(\%)$.

COVID-19, coronavirus disease 2019; HTN, hypertension; COPD, chronic obstructive lung disease; WBC, white blood cell; GFR, glomerular filtration rate. 
$(n=36,30.3 \%)$ and malignancy $(n=23,19.3 \%)$. Most patients (89.9\%) were symptomatic at COVID-19 presentation. During the follow-up period, 34 patients died, and the mortality rate in this study was $28.6 \%$.

Patients who died were older $(75.7 \pm 9.6$ years vs. $59.7 \pm 17.0$ years) and had higher hs-CRP levels $(11.1 \pm 8.6 \mathrm{mg} / \mathrm{dL}$ vs. $5.8 \pm 5.9$ $\mathrm{mg} / \mathrm{dL}, P=0.002)$, and lower GFR $\left(76.7 \pm 20.1 \mathrm{~mL} / \mathrm{min} / 1.73 \mathrm{~m}^{2}\right.$ vs. $\left.95.2 \pm 25.0 \mathrm{~mL} / \mathrm{min} / 1.73 \mathrm{~m}^{2}, P<0.001\right)$ than those who survived. As regards the comorbidities, diabetes (44.1\% vs. $24.7 \%$, $P=0.037)$ and hypertension $(73.5 \%$ vs. $43.5 \%, P=0.003)$ were more prevalent in patients who died than in those who survived.

\section{Thyroid hormone profiles in COVID-19 patients according to disease severity}

The median levels of TSH, T3, and FT4 were $1.18 \mathrm{mIU} / \mathrm{L}, 0.92$ $\mathrm{ng} / \mathrm{mL}$, and $1.24 \mathrm{ng} / \mathrm{dL}$, respectively (Table 2). Of the 119 patients, 76 (63.9\%) were euthyroid and non-presented with overt thyroid dysfunction. NTIS was the most common manifestation (18.5\%) among patients with abnormal thyroid function parameters, followed by subclinical thyrotoxicosis (14.3\%). Of the 17 patients classified as having subclinical thyrotoxicosis, most of them $(16$ of $17,94 \%)$ had mildly suppressed TSH levels ( 0.1 to $0.4 \mathrm{mIU} / \mathrm{L}$ ) and two patients (two of $17,11.8 \%$ ) reported neck pain (Supplemental Table S1).

Of the 119 patients with confirmed COVID-19, 32 had nonsevere disease and 87 had severe to critical disease. Patients with more severe COVID-19 infection had lower TSH and T3 levels. Patients with severe to critical COVID-19 disease had lower TSH (median: $0.90 \mathrm{mIU} / \mathrm{L}$ vs. $1.67 \mathrm{mIU} / \mathrm{L}, P=0.006$ ) and T3 (median: $0.82 \mathrm{ng} / \mathrm{mL}$ vs. $1.11 \mathrm{ng} / \mathrm{mL}, P<0.001$ ) levels compared with those with non-severe disease. Similarly, TSH and T3 levels were significantly lower in non-survivors than in survivors $(P<0.05)$. When classified into diagnostic categories according to thyroid hormone profiles, $81.3 \%$ of the patients with non-severe COVID-19 were euthyroid, whereas $57.5 \%$ of the patients with severe to critical disease and $35.3 \%$ of deceased patients were euthyroid at COVID-19 presentation. NTIS was more commonly observed in patients with severe to critical disease $(23 \%$ vs. $6.3 \%, P=0.037)$ or deceased patients $(38.2 \%$ vs. $11.8 \%, P=0.012$ ) than in those with non-severe disease or in survivors.

\section{Correlation between thyroid hormones and biochemical markers of inflammation}

A significant negative correlation was found between the biochemical markers of inflammation and T3 or TSH levels (Fig. 1). T3 was negatively correlated with hs-CRP $(r=-0.373, P<0.001)$ and WBC count $(r=-0.463, P<0.001)$. TSH was negatively correlated with hs-CRP $(r=-0.224, P=0.014)$; however, it was not correlated with WBC count $(r=-0.126, P=0.172)$.

\section{Correlation between thyroid hormones and the clinical outcome of COVID-19 patients}

The associations between tertiles of thyroid hormones and outcomes of COVID-19 patients are presented in Table 3. COVID-19 patients in the lowest tertile of T3 levels had significantly

Table 2. Comparison of Thyroid Function Test According to the Severity of Disease and Mortality

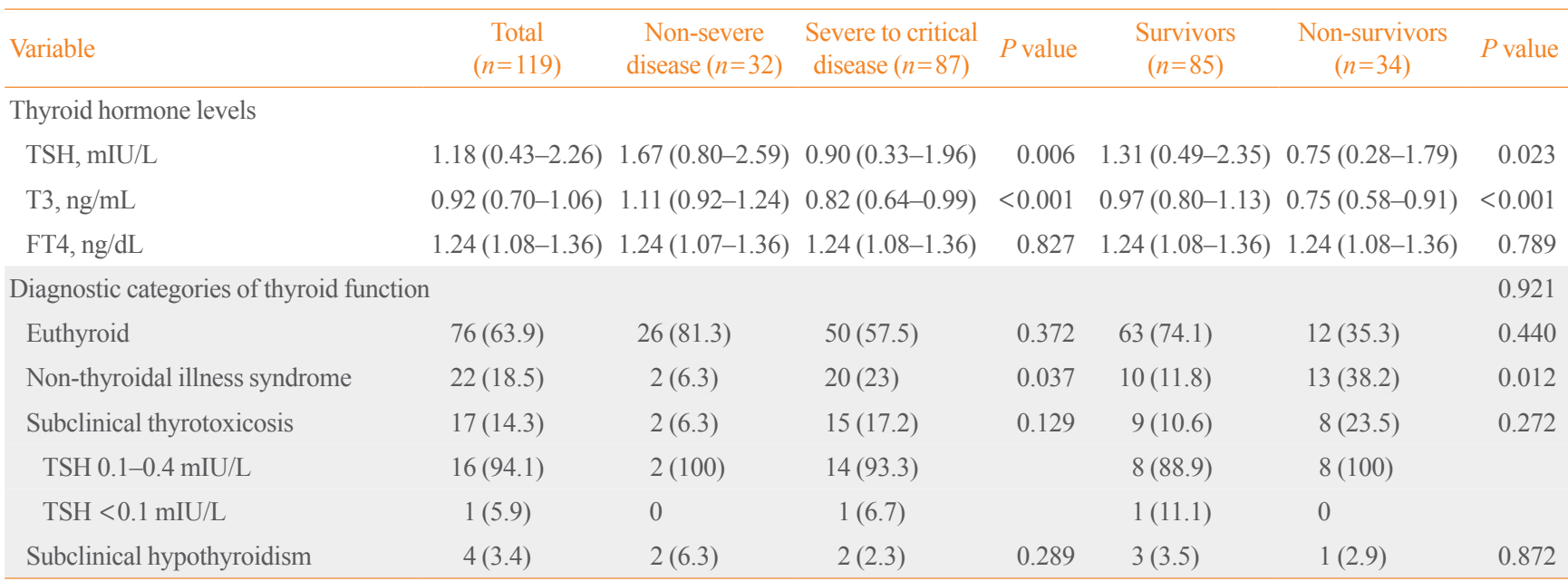

Values are expressed as median (interquartile range) or number (\%).

$\mathrm{TSH}$, thyroid stimulating hormone; T3, triiodothyronine; FT4, free thyroxine. 

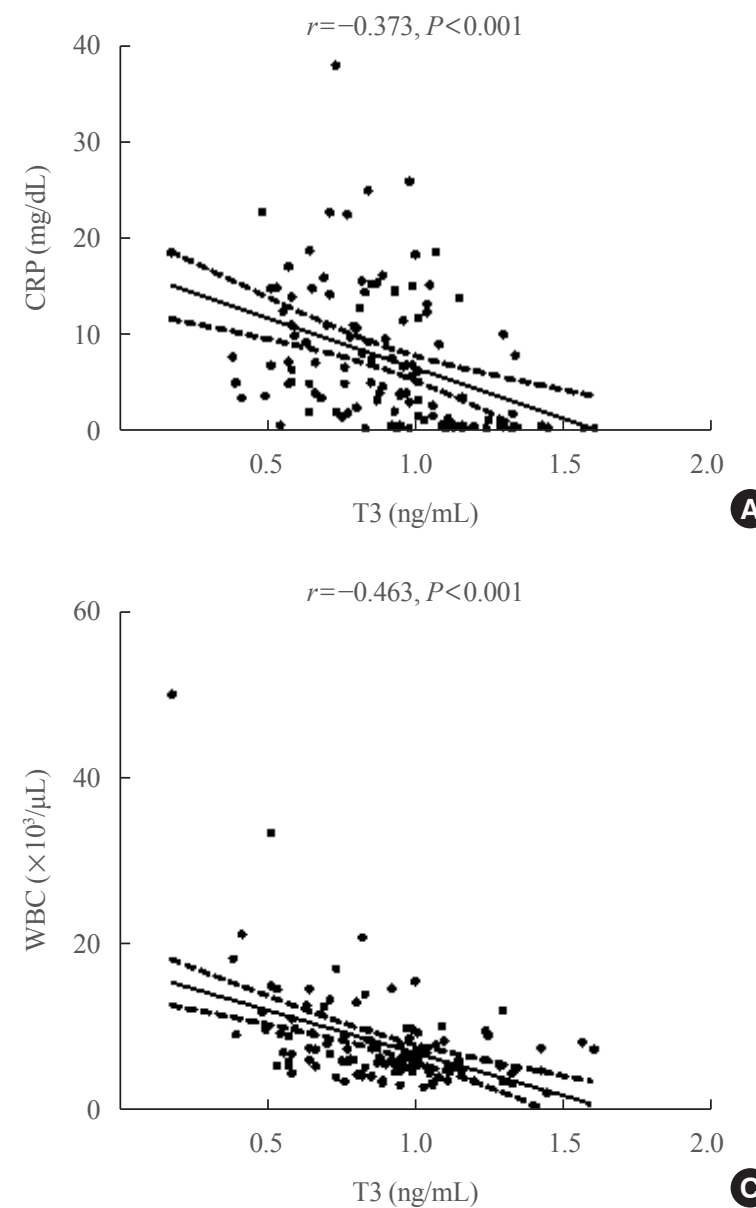
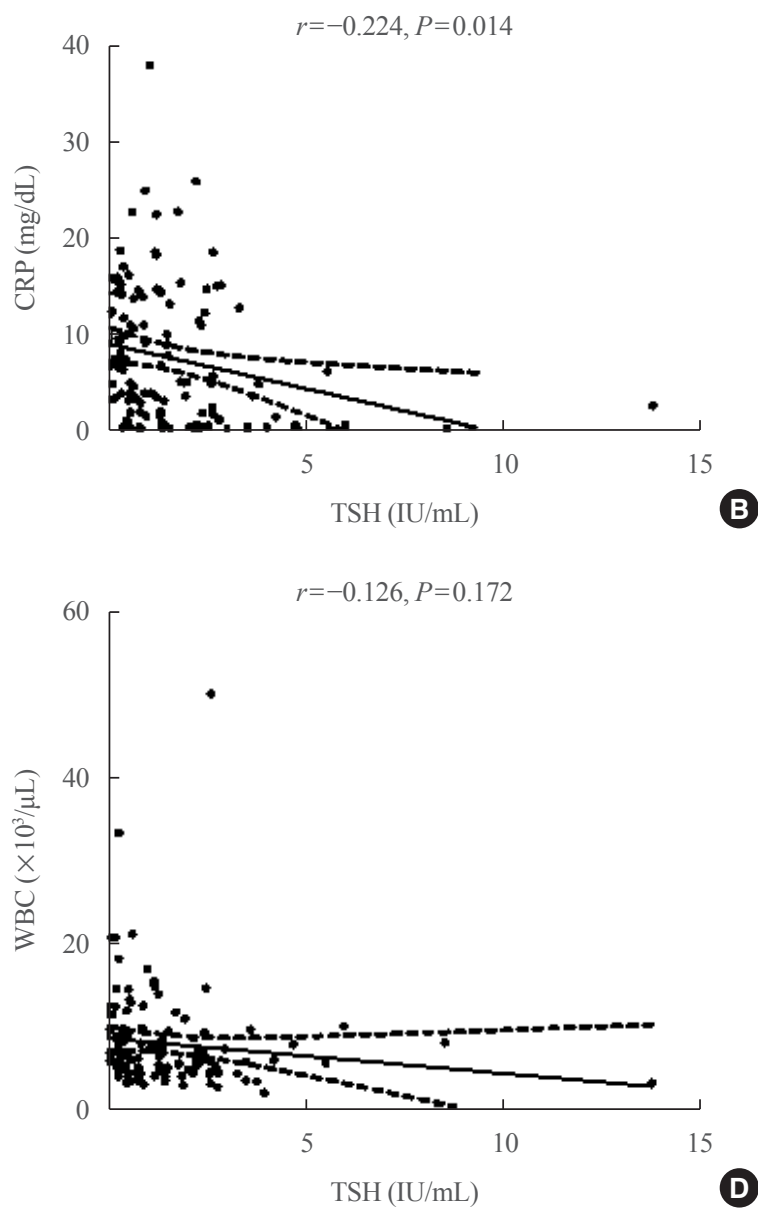

Fig. 1. Scatterplots of thyroid hormones and C-reactive protein (CRP) or white blood cell (WBC). The Pearson correlation coefficient is shown at the top with its associated $P$ value. The regression line (solid line) and 95\% confidence interval (dotted line) are plotted. (A) Correlation between triiodothyronine (T3) and CRP. (B) Correlation between thyroid stimulating hormone (TSH) and CRP. (C) Correlation between T3 and WBC count. (D) Correlation between TSH and WBC count.

Table 3. Outcome of COVID-19 Patients According to Thyroid Hormone Levels

\begin{tabular}{|c|c|c|c|c|}
\hline Clinical outcome & Lowest tertile & Middle tertile & Highest tertile & $P$ trend \\
\hline T3 levels, ng/mL & $\leq 0.77, n=39$ & $\geq 0.78$ and $<0.99, n=40$ & $\geq 1.00, n=40$ & \\
\hline ICU admission & $24(61.5)$ & $13(32.5)$ & $12(30)$ & 0.005 \\
\hline Mechanical ventilation & $18(46.2)$ & $11(27.5)$ & $5(12.5)$ & 0.001 \\
\hline Mortality & $19(48.7)$ & $13(32.5)$ & $2(5)$ & $<0.001$ \\
\hline TSH levels, mIU/L & $\leq 0.56, n=39$ & $\geq 0.57$ and $<1.72, n=40$ & $\geq 1.73, n=40$ & \\
\hline ICU admission & $20(51.3)$ & $15(37.5)$ & $14(35)$ & 0.144 \\
\hline Mechanical ventilation & $20(51.3)$ & $8(20)$ & $6(15)$ & $<0.001$ \\
\hline Mortality & $15(38.5)$ & $11(27.5)$ & $8(20)$ & 0.071 \\
\hline FT4 levels, ng/dL & $\leq 1.14, n=39$ & $\geq 1.15$ and $<1.31, n=40$ & $\geq 1.31, n=40$ & \\
\hline ICU admission & $14(35.9)$ & $21(52.5)$ & $14(35)$ & 0.850 \\
\hline Mechanical ventilation & $9(23.7)$ & $15(37.5)$ & $10(25)$ & 0.915 \\
\hline Mortality & $11(28.2)$ & $12(30)$ & $11(27.5)$ & 0.887 \\
\hline
\end{tabular}

Values are expressed as number $(\%)$.

COVID-19, coronavirus disease 2019; T3, triiodothyronine; ICU, intensive care unit; TSH, thyroid stimulating hormone; FT4, free thyroxine. 


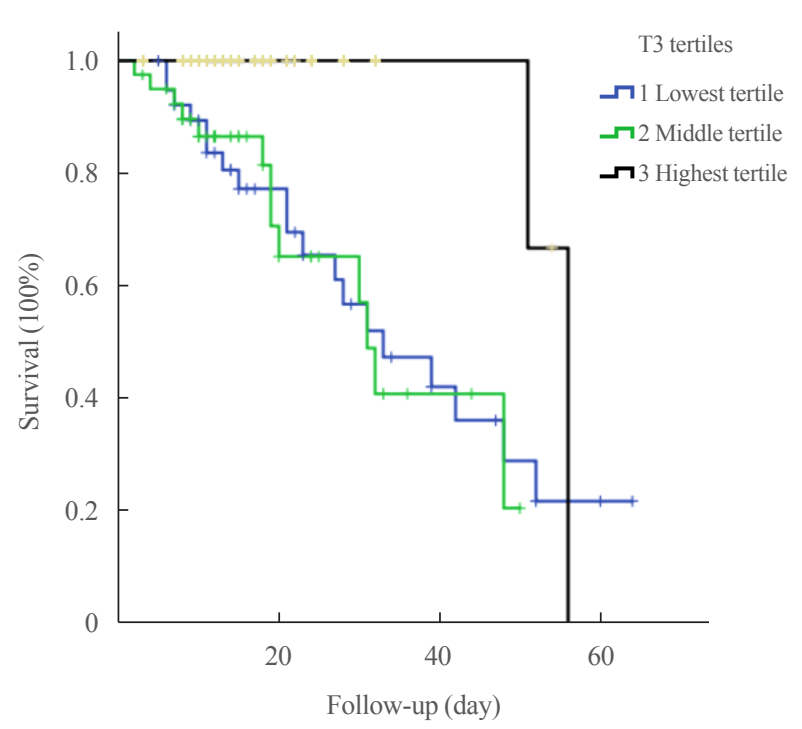

Fig. 2. Kaplan-Meier curves for in-hospital mortality according to triiodothyronine (T3) tertiles.

higher rates of critical events, including ICU admission (61.5\% vs. $32.5 \%$ vs. $30 \%, P$ for trend $=0.005)$, mechanical ventilation $(46.2 \%$ vs. $27.5 \%$ vs. $12.5 \%, P$ for trend $=0.001)$, and death $(48.7 \%$ vs. $32.5 \%$ vs. $5 \%, P$ for trend $<0.001)$ than those in the middle or higher tertile of T3 levels. The Kaplan-Meier curves for survival (Fig. 2) showed that increased mortality of the lowest T3 tertile ( $\log$-rank $P=0.014)$.

Patients in the lowest tertile of TSH also tended to have a higher rate of requiring mechanical ventilation than those in the middle or highest tertiles of TSH levels $(P$ for trend $<0.001)$. No significant association was observed between tertiles of FT4 levels and the clinical outcomes.

We performed a multivariable analysis to determine whether lower $\mathrm{T} 3$ levels independently predicted the all-cause mortality of COVID-19 (Table 4). Lowest T3 level $(<0.77 \mathrm{ng} / \mathrm{mL})$ was independently associated with COVID-19-related mortality (adjusted HR, 5.27; $95 \%$ CI, 1.09 to 25.32; $P=0.038$ ), compared with the highest $\mathrm{T} 3$ level $(>1.00 \mathrm{ng} / \mathrm{mL})$.

\section{DISCUSSION}

In the present study, we investigated the thyroid hormone levels and their prognostic impact in patients with COVID-19. NTIS is the most common type of thyroid dysfunction, followed by subclinical thyrotoxicosis in COVID-19 patients. Changes in TSH and T3 levels were significantly correlated with the severity of COVID-19. Lower T3 levels were associated with COVID-19-related adverse outcomes.

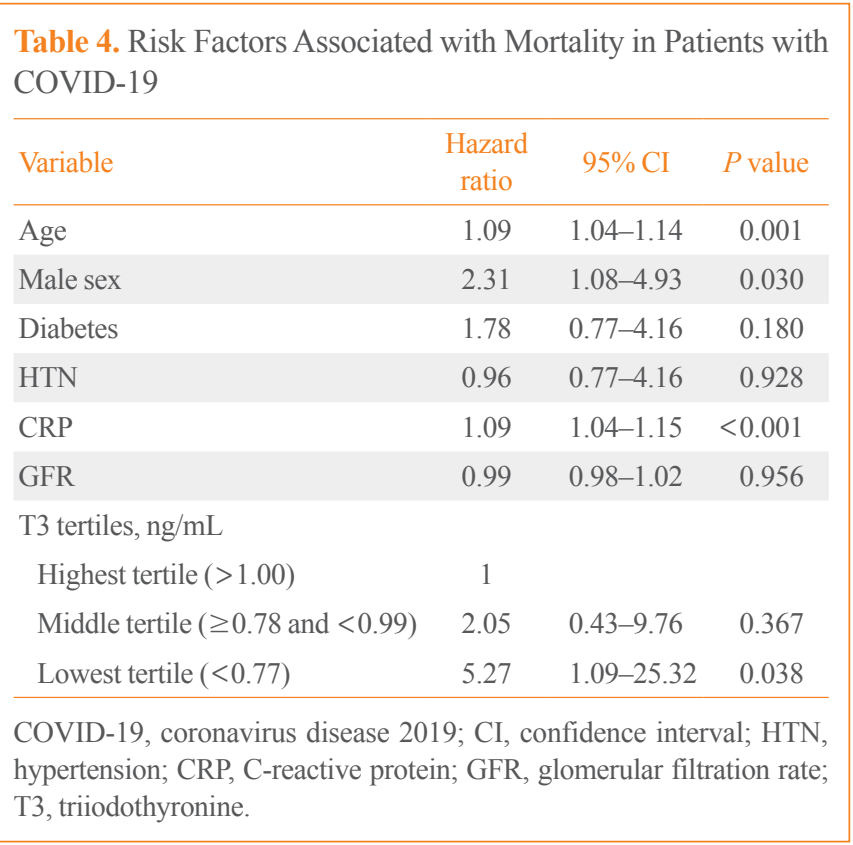

In our study, $63 \%$ of the patients were euthyroid at COVID-19 presentation, and NTIS (18.5\%) was the most common manifestation among patients with abnormal thyroid function. With the spread of COVID-19 worldwide, studies have shown that the clinical manifestations of thyroid involvement in COVID-19 patients are inconsistent [4-12]. Several case reports have described patients with typical subacute thyroiditis after COVID-19 infection $[4,6,8]$. Most of them suffered with mild or moderate COVID-19 disease, none of them experienced ICU admission. Further studies in hospitalized patients found that occurrence of an atypical painless thyroiditis in COVID-19 patients [5,7]. Lania et al. [5] reported overt thyrotoxicosis in 31 (10.8\%) of the 287 patients with COVID-19 who were treated in non-ICU but measured the T3 and T4 levels in only $25 \%$ of patients. Eight patients underwent thyroid ultrasound and none of them had classic ultrasound findings of subacute thyroiditis. Muller et al. [7] reported overt thyrotoxicosis in $15 \%$ of Italian patients with COVID-19 admitted in a high-dependency ICU, compared with $2 \%$ of those treated in low-intensity settings; they defined overt thyrotoxicosis as TSH less than $0.28 \mathrm{mU} / \mathrm{L}$ and/or FT4 greater than 21.9 pmol/L. However, FT4 was measured in only $24 \%$ of the patients. Thyroid ultrasound was performed in five patients with thyrotoxicosis, focal hypoechoic area typical of subacute thyroiditis were observed in three patients. Unlike these two studies, other investigators suggested that the changes in thyroid function in hospitalized COVID-19 patients were more consistent with the NTIS pattern, initially 
characterized by a reduction in total T3 and FT3 levels and normal or slightly decreased TSH levels $[9-12,14]$. In a study conducted in 50 Chinese patients with COVID-19, Chen et al. [9] reported a generalized reduction in $\mathrm{TSH}, \mathrm{T} 3$, and $\mathrm{T} 4$ levels. They also found that the TSH levels were lower than the reference range in $56 \%$ of the patients. In a multicenter study in the United States, Khoo et al. [10] serially assessed the thyroid function of COVID-19 patients at admission and follow-up. They reported that COVID-19 patients had slightly lower levels of TSH and FT4 than those without COVID-19. Furthermore, they found that the thyroid function of COVID-19 survivors returned to baseline at follow-up. In our study, NTIS was more commonly observed in patients with severe to critical disease $(23 \%)$ than in those with non-severe disease $(6.3 \%)$. The frequency of NTIS in our cohort is comparable to those reported in the recent studies, which were $20 \%$ to $30 \%$ among patients with severe COVID-19 [11,12] and higher than that among patients with mild to moderate baseline severity [24]. These findings suggested that alterations in thyroid function at COVID-19 presentation were more similar to an NTIS pattern than to a classic subacute thyroiditis, especially in the patients with more severe COVID-19 disease.

The pathophysiology and expression of NTIS differ depending on the type of illness, disease severity, and the stages of illness [15]. In the acute phase of illness, the plasma concentrations of T3 decreased very rapidly and those of reverse T3 increased. These alterations may partly be explained by the reduced levels of thyroid hormone-binding protein and albumin and reduced binding activity [25]. In addition, an acute alteration in the peripheral conversion of T4 due to the decrease in type 1 deiodinase (D1) activity and increase in type 3 deiodinase (D3) activity may explain these changes [26]. Circulating cytokines are known to decrease the D1 activity and increase the D3 activity, leading to acute fall in T3 levels in critically ill patients [27,28]. In COVID-19, the course and severity of disease are closely related to the actions of several cytokines, including interleukin-6 and tumor necrosis factor alpha [29]. Because cytokine storms commonly occur in patients with COVID-19 [30], the expression of cytokines induced by SARS$\mathrm{CoV}-2$ infection may play an important role in the development of NTIS in COVID-19 patients. In our study, a significant correlation was found between inflammatory markers and TSH/T3 levels. Considering that CRP is well established as a marker of systemic inflammation and severe infection, the degree of changes in thyroid function might be closely associated with a more severe inflammatory response in COVID-19.
In our study, subclinical thyrotoxicosis was observed in $14.5 \%$ of the patients, most of whom had mildly suppressed TSH levels ( 0.1 to $0.4 \mathrm{mIU} / \mathrm{L})$. None of the patients presented with overt thyrotoxicosis suggesting classic subacute thyroiditis, even in patients who died. The suppression of TSH levels might be related to the elevated levels of various proinflammatory cytokines and endogenous cortisol secretion caused by viral infection $[31,32]$. It may also be due to the direct cytopathic effect of SARS-CoV-2 infection on thyrotrophs in the pituitary gland. Coronaviruses have been found in the pituitary gland of a deceased patient, and reduced staining for TSH has been observed in the anterior pituitary gland of patients infected with SARS-CoV [33]. Suppressed TSH levels may be a manifestation of atypical viral subacute thyroiditis. However, this was not confirmed because of the absence of radiologic evaluations, although two of 17 patients with suppressed TSH levels complained of neck pain, in our study.

Identification of the risk factors for COVID-19-related adverse outcomes is important in the setting of a pandemic burden. Previous studies have consistently reported that older age, higher levels of inflammatory parameters including CRP, higher levels of tissue markers, higher levels of fasting glucose, and worse symptoms were associated with poor clinical outcomes in COVID-19 patients [34-37]. In addition, changes in thyroid hormones, especially low T3 levels, have also been suggested as potential prognostic markers in COVID-19 patients [11,13, 14]. In our study, the degree of change in the NTIS seemed to be associated with disease severity and prognosis. Patients with severe to critical disease had significantly lower levels of TSH and T3 than those with non-severe disease. Furthermore, patients with the lowest T3 tertile had COVID-19-related adverse outcomes and were independently associated with a higher risk of mortality, which was similar to the findings of recent studies $[11,13,14]$. Zou et al. [11] reported that NTIS was significantly associated with inflammatory parameters and the severity of COVID-19 disease; however, the mortality rate was not different between patients with NTIS and those without NTIS. Other studies have shown that lower FT3 levels are independently associated with COVID-19-related mortality $[13,14]$. Therefore, NTIS appears to be useful as a potential marker for predicting prognosis in COVID-19.

This study has several limitations. First, thyroid hormone profiles were assessed only at admission because of the retrospective collection of data. Second, additional examinations, including measurement of thyroid autoantibodies and neck ultrasound, were not performed in most patients, because they were not 
usually referred to an endocrinologist and were isolated. Therefore, the etiology of subclinical thyrotoxicosis remains unknown. Third, the FT3 and rT3 levels were not evaluated in this study. However, our findings suggest that T3 levels can serve as a useful prognostic marker in COVID-19.

In conclusion, NTIS was commonly observed in patients with predominantly baseline severe manifestations. The degree of decline in TSH and T3 levels was significantly correlated with the severity of COVID-19. Low T3 levels had prognostic significance and were associated with more adverse COVID19-related outcomes.

\section{CONFLICTS OF INTEREST}

No potential conflict of interest relevant to this article was reported.

\section{AUTHOR CONTRIBUTIONS}

Conception or design: M.K.L., S.Y.S. Acquisition, analysis, or interpretation of data: J.A., S.Y.S. Drafting the work or revising: J.A., S.Y.S. Final approval of the manuscript: J.H.L., S.Y.S.

\section{ORCID}

Jiyeon Ahn https://orcid.org/0000-0002-5957-3394

Seo Young Sohn https://orcid.org/0000-0003-2033-6671

\section{REFERENCES}

1. World Health Organization. Coronavirus disease (COVID-19) outbreak [Internet]. Geneva: WHO; 2021 [cited 2021 Jul 24]. Available from: https://www.who.int/emergencies/ diseases/novel-coronavirus-2019.

2. Zhu N, Zhang D, Wang W, Li X, Yang B, Song J, et al. A novel coronavirus from patients with pneumonia in China, 2019. N Engl J Med 2020;382:727-33.

3. Li MY, Li L, Zhang Y, Wang XS. Expression of the SARSCoV-2 cell receptor gene ACE2 in a wide variety of human tissues. Infect Dis Poverty 2020;9:45.

4. Brancatella A, Ricci D, Cappellani D, Viola N, Sgro D, Santini $\mathrm{F}$, et al. Is subacute thyroiditis an underestimated manifestation of SARS-CoV-2 infection? Insights from a case series. J Clin Endocrinol Metab 2020;105:dgaa537.

5. Lania A, Sandri MT, Cellini M, Mirani M, Lavezzi E, Mazziotti G. Thyrotoxicosis in patients with COVID-19: the
THYRCOV study. Eur J Endocrinol 2020;183:381-7.

6. Ruggeri RM, Campenni A, Siracusa M, Frazzetto G, Gullo D. Subacute thyroiditis in a patient infected with SARSCOV-2: an endocrine complication linked to the COVID-19 pandemic. Hormones (Athens) 2021;20:219-21.

7. Muller I, Cannavaro D, Dazzi D, Covelli D, Mantovani G, Muscatello A, et al. SARS-CoV-2-related atypical thyroiditis. Lancet Diabetes Endocrinol 2020;8:739-41.

8. Asfuroglu Kalkan E, Ates I. A case of subacute thyroiditis associated with COVID-19 infection. J Endocrinol Invest 2020;43:1173-4.

9. Chen M, Zhou W, Xu W. Thyroid function analysis in 50 patients with COVID-19: a retrospective study. Thyroid 2021; 31:8-11.

10. Khoo B, Tan T, Clarke SA, Mills EG, Patel B, Modi M, et al. Thyroid function before, during, and after COVID-19. J Clin Endocrinol Metab 2021;106:e803-11.

11. Zou R, Wu C, Zhang S, Wang G, Zhang Q, Yu B, et al. Euthyroid sick syndrome in patients with COVID-19. Front Endocrinol (Lausanne) 2020;11:566439.

12. Zhang Y, Lin F, Tu W, Zhang J, Choudhry AA, Ahmed O, et al. Thyroid dysfunction may be associated with poor outcomes in patients with COVID-19. Mol Cell Endocrinol 2021; 521:111097.

13. Schwarz Y, Percik R, Oberman B, Yaffe D, Zimlichman E, Tirosh A. Sick euthyroid syndrome on presentation of patients with COVID-19: a potential marker for disease severity. Endocr Pract 2021;27:101-9.

14. Gao W, Guo W, Guo Y, Shi M, Dong G, Wang G, et al. Thyroid hormone concentrations in severely or critically ill patients with COVID-19. J Endocrinol Invest 2021;44:1031-40.

15. Van den Berghe G. Non-thyroidal illness in the ICU: a syndrome with different faces. Thyroid 2014;24:1456-65.

16. Su W, Zhao XQ, Wang M, Chen H, Li HW. Low T3 syndrome improves risk prediction of in-hospital cardiovascular death in patients with acute myocardial infarction. J Cardiol 2018;72:215-9.

17. Todd SR, Sim V, Moore LJ, Turner KL, Sucher JF, Moore FA. The identification of thyroid dysfunction in surgical sepsis. J Trauma Acute Care Surg 2012;73:1457-60.

18. Guo J, Hong Y, Wang Z, Li Y. Prognostic value of thyroid hormone FT3 in general patients admitted to the intensive care unit. Biomed Res Int 2020;2020:6329548.

19. Angelousi AG, Karageorgopoulos DE, Kapaskelis AM, Falagas ME. Association between thyroid function tests at baseline and the outcome of patients with sepsis or septic shock: 
a systematic review. Eur J Endocrinol 2011;164:147-55.

20. Wu Y, You S, Zang H, Liu H, Mao Y, Mao P, et al. Usefulness of serum thyroid-stimulation hormone (TSH) as a prognostic indicator for acute-on-chronic liver failure. Ann Hepatol 2015;14:218-24.

21. Ceresini G, Marina M, Lauretani F, Maggio M, Bandinelli S, Ceda GP, et al. Relationship between circulating thyroidstimulating hormone, free thyroxine, and free triiodothyronine concentrations and 9-year mortality in euthyroid elderly adults. J Am Geriatr Soc 2016;64:553-60.

22. Lee KD, Lee SB, Lim JK, Kang YM, Kim IB, Moon HJ, et al. Providing essential clinical care for non-COVID-19 patients in a Seoul metropolitan acute care hospital amidst ongoing treatment of COVID-19 patients. J Hosp Infect 2020; 106:673-7.

23. China National Health Commission. Chinese clinical guidance for COVID-19 pneumonia diagnosis and treatment (7th edition) [Internet]. Beijing: Chinese Society of Cardiology; 2020 [cited $2021 \mathrm{Jul}$ 24]. Available from: http://kjfy.meetingchina. org/msite/news/show/cn/3337.html.

24. Lui DTW, Lee CH, Chow WS, Lee ACH, Tam AR, Fong $\mathrm{CHY}$, et al. Role of non-thyroidal illness syndrome in predicting adverse outcomes in COVID-19 patients predominantly of mild-to-moderate severity. Clin Endocrinol (Oxf) 2021 Apr 4 [Epub]. https://doi.org/10.1111/cen.14476.

25. Docter R, van Toor H, Krenning EP, de Jong M, Hennemann G. Free thyroxine assessed with three assays in sera of patients with nonthyroidal illness and of subjects with abnormal concentrations of thyroxine-binding proteins. Clin Chem 1993;39:1668-74.

26. Peeters RP, Wouters PJ, Kaptein E, van Toor H, Visser TJ, Van den Berghe G. Reduced activation and increased inactivation of thyroid hormone in tissues of critically ill patients. J Clin Endocrinol Metab 2003;88:3202-11.

27. Monig H, Arendt T, Meyer M, Kloehn S, Bewig B. Activation of the hypothalamo-pituitary-adrenal axis in response to septic or non-septic diseases: implications for the euthyroid sick syndrome. Intensive Care Med 1999;25:1402-6.

28. Wajner SM, Goemann IM, Bueno AL, Larsen PR, Maia AL. IL-6 promotes nonthyroidal illness syndrome by blocking thyroxine activation while promoting thyroid hormone inactivation in human cells. J Clin Invest 2011;121:1834-45.

29. McGonagle D, Sharif K, O’Regan A, Bridgewood C. The role of cytokines including interleukin-6 in COVID-19 induced pneumonia and macrophage activation syndrome-like disease. Autoimmun Rev 2020;19:102537.

30. Ye Q, Wang B, Mao J. The pathogenesis and treatment of the 'cytokine storm' in COVID-19. J Infect 2020;80:607-13.

31. Tan T, Khoo B, Mills EG, Phylactou M, Patel B, Eng PC, et al. Association between high serum total cortisol concentrations and mortality from COVID-19. Lancet Diabetes Endocrinol 2020;8:659-60.

32. Torpy DJ, Tsigos C, Lotsikas AJ, Defensor R, Chrousos GP, Papanicolaou DA. Acute and delayed effects of a singledose injection of interleukin-6 on thyroid function in healthy humans. Metabolism 1998;47:1289-93.

33. Wei L, Sun S, Zhang J, Zhu H, Xu Y, Ma Q, et al. Endocrine cells of the adenohypophysis in severe acute respiratory syndrome (SARS). Biochem Cell Biol 2010;88:723-30.

34. Smilowitz NR, Kunichoff D, Garshick M, Shah B, Pillinger M, Hochman JS, et al. C-reactive protein and clinical outcomes in patients with COVID-19. Eur Heart J 2021;42: 2270-9.

35. Li X, Xu S, Yu M, Wang K, Tao Y, Zhou Y, et al. Risk factors for severity and mortality in adult COVID-19 inpatients in Wuhan. J Allergy Clin Immunol 2020;146:110-8.

36. Wang Z. Identification of risk factors for in-hospital death of COVID-19 pneumonia-lessions from the early outbreak. BMC Infect Dis 2021;21:113.

37. Chang MC, Hwang JM, Jeon JH, Kwak SG, Park D, Moon JS. Fasting plasma glucose level independently predicts the mortality of patients with coronavirus disease 2019 infection: a multicenter, retrospective cohort study. Endocrinol Metab (Seoul) 2020;35:595-601. 